\title{
Nonautonomous Hamiltonians
}

\author{
A. Soffer ${ }^{1}$ and M. I. Weinstein ${ }^{2,3}$
}

Received January 13, 1998

\begin{abstract}
We present a theory of resonances for a class of nonautonomous Hamiltonians to treat the structural instability of spatially localized and time-periodic solutions associated with an unperturbed autonomous Hamiltonian. The mechanism of instability is radiative decay, due to resonant coupling of the discrete modes to the continuum modes by the time-dependent perturbation. This results in a slow transfer of energy from the discrete modes to the continuum. The rate of decay of solutions is slow and hence the decaying bound states can be viewed as metastable. The ideas are closely related to the authors' work on (i) a timedependent approach to the instability of eigenvalues embedded in the continuous spectra, and (ii) resonances, radiation damping, and instability in Hamiltonian nonlinear wave equations. The theory is applied to a general class of Schrödinger equations. The phenomenon of ionization may be viewed as a resonance problem of the type we consider and we apply our theory to find the rate of ionization, spectral line shift, and local decay estimates for such Hamiltonians.
\end{abstract}

KEY WORDS: Resonance; lifetime; ionization; breather.

\section{INTRODUCTION}

There are many dynamical systems of interest for which the dynamics can be viewed as given by a Schrödinger type equation generated by an operator $H(t)=H_{0}+W(t)$. Here, $H_{0}$ is a time-independent (autonomous) part, assumed to be self-adjoint on a complex Hilbert space $\mathscr{H}$, and $W(t)$ denotes time-dependent (non-autonomous) perturbation. We consider the initial value problem for the Schrödinger equation for $\phi: t \mapsto \phi(t) \in \mathscr{H}$ :

$$
i \partial_{t} \phi=\left(H_{0}+W(t)\right) \phi
$$

${ }^{1}$ Department of Mathematics, Rutgers University, New Brunswick, New Jersey.

${ }^{2}$ Department of Mathematics, University of Michigan, Ann Arbor, Michigan.

${ }^{3}$ Mathematical Sciences Research, Bell Labs 2c-358, Murray Hill, New Jersey. 
with initial condition $\phi(0)=\phi_{0}$ in a suitable subspace of $\mathscr{H}$. An important special case, studied in Section 3, is:

$$
i \partial_{t} \phi(t, x)=\left(H_{0}+W(t, x)\right) \phi(t, x)
$$

where $H_{0}=-\Delta+V(x)$, with $V(x)$ real-valued and $W(t, x)$ is a smooth function which is spatially localized and time-periodic or quasi-periodic. Equations of this type play an important role in tunneling and ionization physics, and are relevant to an understanding of many nonlinear problems. This is discussed later in this section.

In this paper, we consider the situation where the unperturbed equation, corresponding to the case $W \equiv 0$, has a time-periodic solution in $\mathscr{H}$, e.g., a bound state. We study the structural instability of the bound state due to a perturbation $W(t)$, which is assumed to be periodic. The analysis is carried out for the special case $W(t)=\cos (\mu t) \beta$, with $\beta^{*}=\beta$; see Section 2 . The case of more general perturbations $W(t)$, which are periodic or quasiperiodic, as well as the case when the unperturbed operator $H_{0}$ has more than bound state can be treated by our approach. We indicate briefly in Section 4 the modifications required in the analysis.

Viewed in terms of the coordinates of the unperturbed dynamical system, the perturbation $W(t)$ has the effect of coupling the bound state to the continuum modes. The mechanism of instability is a slow transfer of energy from the bound state part of the solution to the continuum modes and an associated decay due to radiation of energy to infinity. We develop a theory which yields a detailed picture of the intermediate and long time behavior of solutions to the initial value problem. Our main results are presented in Theorems 2.1 and 2.2. A consequence is that the solution of the initial value problem for the perturbed dynamical system with initial data given by the time-periodic bound state of the unperturbed problem, is characterized by transient exponential decay $\sim e^{-\Gamma|t|}$ where $0<\Gamma=$ $\mathcal{O}\left(\|W\|^{2}\right)$, for $\|W\|||$ small, and by algebraic decay as $|t| \rightarrow \infty$. We derive explicit formulae for the lifetime, $\tau \equiv \Gamma^{-1}$ (Eq. (2.9)) and the spectral line shift (Eq. (4.21)).

The approach we take is closely related to our work on quantum resonances ${ }^{(37,38)}$ and on the radiation damping of localized states for nonlinear wave equations. ${ }^{(39)}$ We now illustrate the main idea in the current context. Consider a linear Schrödinger equation:

$$
i \partial_{t} \phi(t, x)=(-\Delta+V(x)) \phi(t, x)+\cos (\mu t) \beta(x) \phi(t, x)
$$

We assume that $-\Delta+V(x)$ has exactly one eigenvalue at $\lambda_{0}<0$, with corresponding eigenfunction $\psi_{0}(x)$. Therefore, the unperturbed $(\beta \equiv 0)$ 
problem has a spatially localized and time-periodic solution, $\psi_{0}(x) e^{-i \dot{\lambda}_{0} t}$. We assume that the continuous spectrum of $H_{0}$ is equal to the real nonnegative half-line. The perturbation $W(x, t)=\beta(x) \cos (\mu t)$ is assumed to be real-valued, to decay sufficiently rapidly as $|x| \rightarrow \infty$, and to be small in an appropriate norm; see Section 3.

We now ask the following question: Does the bound state solution of the unperturbed problem persist under small perturbations of this type?

As indicated above, we find that generic perturbations lead to a slow radiative decay of solutions. To illustrate the mechanism of decay, we first observe that it is natural in the unperturbed case to decompose the solution as:

$$
\phi(t, x)=a(t) \psi_{0}+\phi_{d}(t, x), \quad\left(\psi_{0}, \phi_{d}(t)\right)=0
$$

Then, we have that

$$
\begin{aligned}
i \partial_{t} a(t) & =\lambda_{0} a(t) \\
i \phi_{d}(t, x) & =(-A+V(x)) \phi_{d}(t, x)
\end{aligned}
$$

with initial conditions

$$
a(0)=\left(\psi_{0}, \phi(0)\right), \quad \phi_{d}(0)=\mathbf{P}_{\mathbf{c}} \phi(0) \equiv \phi(0)-\left(\psi_{0}, \phi(0)\right) \psi_{0}
$$

$\mathbf{P}_{\mathbf{c}}$ is the projection onto the continuous spectral part of the operator $H_{0}$. The equations (1.5) decouple and we have that all solutions of (1.5) are of the form (1.4) with: (i) $a(t)=e^{-i \lambda_{0} t} a(0)$, and under suitable hypotheses on $V(x)$, (ii) $\phi_{d}(t, x)$ decays (dispersively) as $|t| \rightarrow \infty$, in the sense that $\left\|\phi_{d}(t, \cdot)\right\|_{p} \rightarrow 0,(p>2)$ or for some $\sigma>0,\left\|\langle x\rangle^{-\sigma} \phi_{d}(t, \cdot)\right\|_{L^{2}} \rightarrow 0$.

How is the dynamics effected by a small spatially localized and timeperiodic perturbation $(\beta \neq 0)$ ? Substitution of $(1.4)$ into (1.3), leads to a weakly coupled dynamical system for $a(t)$ and $\phi_{d}(t)$. This system is derived and studied in Sections 4-6. A simplification of the resulting system which we shall use to illustrate the main idea is the following:

$$
\begin{aligned}
i \partial_{t} a(t) & =\lambda_{0} a(t)+\left(\beta \psi_{0}, \phi_{d}(t)\right) \cos (\mu t) \\
i \partial_{t} \phi_{d}(t) & =-\Delta \phi_{d}(t)+a(t) \cos (\mu t) \beta \psi_{0}
\end{aligned}
$$

The system (1.7) can viewed as an infinite dimensional dynamical system consisting of two components: (i) a finite dimensional part, describing an "oscillation" with complex amplitude, $a(t)$, coupled to (ii) an 
infinite dimensional dispersive wave equation on $\mathbb{R}^{n}$, governing $\phi_{d}(t, x)$. It is simple to check that the system (1.7) has the conserved energy:

$$
\mathscr{E}\left[a, \phi_{d}\right](t) \equiv|a(t)|^{2}+\int\left|\phi_{d}(t, x)\right|^{2} d x=\mathscr{E}\left[a(0), \phi_{d}(0)\right]
$$

The first term in (1.8) corresponds to the oscillator energy and the second to the wave energy.

Intuitively, a spatially localized finite energy disturbance of the rest state should result in motions induced in the oscillator as well as waves propagating "outward" and eventually escaping any compact set (a characteristic of dispersive waves). Due to the coupling $(\beta \neq 0)$ of the oscillator and waves, we expect the energy of the oscillator, $|a(t)|^{2}$, to decay toward zero as time progresses. This energy is transferred to the continuum modes, while the total energy of the system, $\mathscr{E}[a, u]$, remains constant in time. This is what is proved under suitable hypotheses in Theorems 2.1 and 2.2.

The way in which we establish energy transfer from the discrete to the continuum modes is by showing that one can transform a system of the type (1.7) to a normal form, in which internal damping, which drives the energy transfer, is made explicit. This damping is due to a resonant coupling of the discrete oscillator mode with the continuum modes. We now explain the origin of this effect.

Viewing $\beta$ as small, we set $a(t)=e^{-i \lambda_{0} t} A(t)$, where $A(t)$ is "slowly varying," $\left(\partial_{t} A(t)=\mathcal{O}(\beta)\right)$. In terms of the amplitude, $A(t)$, the system (1.7) becomes:

$$
\begin{aligned}
\partial_{t} A(t) & =-i\left(\beta \psi_{0}, \phi_{d}(t)\right) \cos \mu t e^{i \lambda_{0} t} \\
i \partial_{t} \phi_{d} & =-\Delta \phi_{d}+A(t) e^{-i \lambda_{0} t} \cos (\mu t) \beta \psi_{0}
\end{aligned}
$$

Since the variation of $A(t)$ is slow, the source term in (1.10) is essentially quasi-periodic in time. One of its frequencies is $\lambda_{0}+\mu$, which we assume to be strictly positive, i.e., $\lambda_{0}+\mu \in \sigma_{\text {cont }}(-\Delta)$. That is, the non-autonomous parametric forcing of frequency $\mu$ induces a resonant forcing of the wavefield $\phi_{d}$. The character of solutions to (1.9)-(1.10) can be deduced by first solving (1.10) for $\phi_{d}$ and then substituting the result into (1.9). The result is a reduction to an ordinary differential equation for $A(t)$. This is reminiscent of the center manifold approach, commonly used in dissipative systems (see, for example, refs. 3 and 41) and recently applied in a Hamiltonian context. ${ }^{(16)}$

The asymptotic behavior of the ordinary differential equation governing $A(t)$ can be deduced by a asymptotic evaluation of the right hand side of (1.9), considering carefully the contribution to $\phi_{d}$ coming from a neighborhood of the resonant energy, $\lambda_{0}+\mu$. 


$$
\begin{aligned}
-i\left(\beta \psi_{0}, \phi_{d}(t)\right) \cos \mu t e^{i \lambda_{0} t} \\
=-\frac{i}{4}\left(\beta \psi_{0}, \int_{0}^{t} e^{i \Delta(t-s)} e^{-i\left(\lambda_{0}+\mu\right) s} A(s) d s \beta \psi_{0}\right) e^{i\left(\lambda_{0}+\mu\right) t} \\
\quad+\text { nonresonant terms } \\
=-\frac{1}{4}\left(\beta \psi_{0},\left(-\Delta-\lambda_{0}-\mu-i 0\right)^{-1} \beta \psi_{0}\right) A(t) \\
\quad+\text { nonresonant terms }
\end{aligned}
$$

Substitution of this result into the equation for $A(t)$ yields:

$$
\partial_{1} A(t)=(-\Gamma+i A+\rho(t)) A(t) \quad t>0
$$

where

$$
\Gamma=\pi\left(\beta \psi_{0}, \delta\left(-\Delta-\lambda_{0}-\mu\right) \beta \psi_{0}\right)
$$

$A$ is a real constant (the Lamb shift) and $\mathcal{C}\left(\beta^{2}\right)$ and $\rho(t)$ is a bounded oscillatory function of $t$ which is $\mathscr{C}\left(\beta^{2}\right)$.

The right hand side of (1.13) is defined using the spectral theorem for $-\Delta$ on $L^{2}\left(\mathbb{R}^{n}\right)$. In terms of the Fourier transform, $\overline{\mathscr{F}}[z](\xi)=$ $\int e^{-i \xi \cdot x} z(x) d x$, we find:

$$
\Gamma=\frac{1}{16}\left(\lambda_{0}+\mu\right)^{-1 / 2}\left(\left|\mathscr{\mathscr { F }}\left[\beta \psi_{0}\right]\left(\sqrt{\lambda_{0}+\mu}\right)\right|^{2}+\left|\mathscr{\mathscr { F }}\left[\beta \psi_{0}\right]\left(-\sqrt{\lambda_{0}+\mu}\right)\right|^{2}\right)
$$

The constant $\Gamma$ is always non-negative. If it is strictly positive, then (1.12) is a damped oscillator, with damping coefficient $\Gamma$ resulting from coupling of the discrete and continuum modes. In our general theorems, Theorems 2.1 and 2.2, we have hypothesis (H6), that the analogous general expression for $\Gamma$ is strictly positive. This holds generically in the space of perturbations $W$ under consideration.

\subsection{Emergence of Irreversible Behavior in a Time-Reversible System}

Thus, the original dynamical system, (1.3), which is Hamiltonian and, in particular, time-reversible (invariant under the transformation $\phi(t, x) \mapsto$ $\bar{\phi}(-t, x))$, is equivalent to a dynamical system with some apparent time- 
asymmetry. ${ }^{3}$ This is related to the $\varepsilon$-prescription discussed in Section 4 ; see, in particular, Proposition 4.2. At the heart of this apparent paradox is that the energy of the oscillator, which is coupled to the continuum, is propagated out to infinity because the singular limit:

$$
\lim _{\varepsilon \downarrow 0} e^{-i H_{0} t}\left(-\Delta-\left(\lambda_{0}+\mu\right) \mp i \varepsilon\right)^{-1}
$$

satisfies a local energy decay estimate as $t \rightarrow \pm \infty$; see (H4). Thus, for $t<0$, in (1.12) we replace $\Gamma$ by $-\Gamma$.

\subsection{Normal Forms for Coupled Oscillator-Wave Systems}

The damped oscillator above is a normal form equation, which illustrates a more general phenomenon. Dynamical systems like (1.3) can be viewed as a Hamiltonian systems consisting of two subsystems: a single (or, in general, multiple) degree of freedom oscillator and an infinite dimensional radiating wave field.

(I) In (1.3) the discrete oscillator frequency does not lie in the continuous spectrum of wave frequencies. However, the time periodicity of the potential generates frequencies in the solution which resonate with continuum modes.

(II) In refs. 37 and 38, quantum resonance type problems are considered in which the discrete oscillator frequency coincides with a frequency of continuum modes. Coupling of the two occurs due to a time-independent localized potential.

(III) In our study of radiation damping of "breather-like" structures $^{(39)}$ we find, as in (1.3), that the discrete oscillator frequency does not lie in the continuous spectrum of wave frequencies. Here, the coupling is due to a nonlinear potential which generates frequencies which lie in the continuous spectrum.

\footnotetext{
${ }^{3}$ This is related to a "paradox" raised in the late nineteenth century by Zermelo and others concerning the irreversibility of macroscopically observed behavior in physical systems and the reversibility of the microscopic equations of motion. This was addressed in the context of classical systems via a statistical approach in the work of Maxwell and Thomson and culminated in the work of Boltzmann. In quantum systems, for example in the quantum measurement theory, extension of these ideas apply. The source of the emergence of irreversibility, is the coupling of systems with few degrees of freedom to systems with many. For a discussion, see ref. 20.
} 
In an isolated single degree of freedom Hamiltonian system, the general normal form is:

$$
A^{\prime}=i\left(c_{10}+c_{21}|A|^{2}+c_{32}|A|^{4}+\cdots+c_{n+1, n}|A|^{2 n}+\cdots\right) A
$$

where the constants $c_{n+1, n}$ are real numbers.

How is this normal form altered as a result of coupling of the oscillator to an infinite dimensional radiative wave equation?

In refs. 37, 38, and 39 and in the present work, we find the more general dispersive or radiative Hamiltonian normal form:

$$
A^{\prime}=\left(k_{10}+k_{21}|A|^{2}+k_{32}|A|^{4}+\cdots+k_{n+1, n}|A|^{2 n}+\cdots\right) A
$$

with complex coefficients;

$$
k_{n+1, n}=d_{n+1, n}+i c_{n+1, n}
$$

where $d_{n+1, n}$ and $c_{n+1, n}$ are real numbers. Non-zero real parts of the normal form coefficients arise due to coupling of discrete and continuum modes. In the linear problems: (I) and (II), we always have $d_{10} \equiv-\Gamma \leqslant 0$. In (III) we have $k_{10}=i c_{10}$ and, depending on the details of the nonlinear interaction, we may have: $k_{21}=i c_{21}$ and $k_{32}=-\Gamma+i c_{32},{ }^{\left({ }^{39)}\right.} k_{21}=-\Gamma+$ $i c_{21}{ }^{(26)}$ or other possibilities. In each case there's a different expression for $\Gamma$, the first nontrivial real contribution to a normal form coefficient, but the essence of its calculation is as described above. Generically one has $-\Gamma<0$, and it is natural to conjecture that the first non-zero $\Gamma=d_{n_{*}+1, n_{*}}$ is strictly negative.

\subsection{Connection with Persistence Theory of Periodic Solutions}

The condition $\Gamma>0$ (see also (H6)) can be seen to arise in another manner. Motivated by the perturbative approach to constructing periodic solutions of ordinary differential equations, it is natural to seek a solution of (1.3) in the form:

$$
\phi(t)=e^{-i \lambda_{0} t} \psi_{0}+\psi_{1}+\psi_{2}+\cdots+\psi_{j}+\cdots
$$

where $\psi_{j}$ is formally of order $\beta^{j}$. Substitution of the ansatz (1.19) into (1.3) yields that:

$$
\psi_{1}=\psi_{1}^{a}+\psi_{1}^{b}
$$


where

$$
\begin{aligned}
& i \partial_{t} \psi_{1}^{a}=H_{0} \psi_{1}^{a}+\frac{1}{2} e^{-i\left(\lambda_{0}-\mu\right) t} \beta \psi_{0} \\
& i \partial_{t} \psi_{1}^{b}=H_{0} \psi_{1}^{b}+\frac{1}{2} e^{-i\left(\lambda_{0}+\mu\right) t} \beta \psi_{0}
\end{aligned}
$$

These equations can be solved by setting:

$$
\psi_{1}^{a}=e^{-t\left(\lambda_{0}-\mu\right) t} \psi^{a}, \psi_{1}^{b}=e^{-i\left(\lambda_{0}+\mu\right) t} \psi^{b}
$$

For $\psi_{1}^{a}$ we obtain the equation:

$$
\begin{aligned}
& \left(H_{0}-\left(\lambda_{0}-\mu\right)\right) \Psi^{a}=\frac{1}{2} \beta \psi_{0} \\
& \left(H_{0}-\left(\lambda_{0}+\mu\right)\right) \Psi^{b}=\frac{1}{2} \beta \psi_{0}
\end{aligned}
$$

The first equation of (1.23) has a localized solution because $\lambda_{0}-\mu<0$ and lies in the resolvent set of $H_{0}$. Note however, that $\lambda_{0}+\mu>0$, and therefore the second equation can have a localized solution only if the projection of $\beta \psi_{0}$ onto the generalized eigenfunction associated with the continuum energy $\lambda_{0}+\mu$ vanishes. This is equivalent to $\Gamma=0$, where $\Gamma$ is defined by (1.13); see also (1.14) or, more generally, (H6). Generically, $\Gamma>0$. The results of this paper show, in particular, that this obstruction to solvability in the class of quasiperiodic functions, implies radiative decay of solutions of the initial value problem.

Finally, we discuss various contexts, where problems involving timedependent potentials have been studied. Equations with time dependent potentials arise in mathematical physics and are important in applications, e.g., electron microscopy and solid state devices. In quantum physics their analysis dates back to the early days of the theory, where the process of ionization by external field (light waves) was studied and later nuclear spin resonance and charge transfer problems; see, e.g., refs. 19 and 7. The problem of ionization and more generally of excitation of a molecule by radiation is also important in laser optics and chemistry. Recent models of $\operatorname{chaos}^{(14)}$ and stochastic resonances also involve time dependent potential models, e.g., ref. 42. The expression (1.13) (see also (H6)) is the analogue of the Fermi Golden Rule, which arises in the study of spontaneous emission, autoionization. ${ }^{(7,19,29)}$ In the context of the problem of ionization of an atom by a time-dependent electric field a heuristic derivation is presented in Section 42 of ref. 19.

Equations of type (1.1) and (1.2) are also relevant in the study of coherent solutions of nonlinear systems. For example, a periodic or quasiperiodic solution can be viewed as a bound state of an equation of this type with a self-consistent (nonlinear) time-dependent potential. This point of view was first exploited by Sigal ${ }^{(33,24)}$ who used the methods of 
dilation analyticity to relate the generic structural instability of quasi-periodic solutions of certain nonlinear systems to the generic instability of an embedded eigenvalue in the continuous spectrum of a Floquet operator. This approach yields the spectral line shift and the Fermi golden rule, which give the location of the resonance to which the embedded eigenvalue has been perturbed. The approach we have taken to the non-persistence question is to show by a study of the large time behavior of the initial value problem that solutions decay slowly as $t \rightarrow \infty$.

The analysis of time dependent resonant problems in the physics literature is based on formal time dependent perturbation theory; see refs. 19 and 7, where Dyson series to first order is used, and ref. 30 where Dirac's perturbation theory is applied. This analysis is limited in many respects. In refs. 19 and 7 it is assumed, among other things, that the relevant time scale of the approximation is smaller than $\varepsilon^{-1}$ where $\varepsilon$ is the perturbation size, while we know that the lifetime, is of order $\varepsilon^{-2}$; It is also not clear how to get the spectral line shift from this approach.

Problems involving time dependent potentials have received considerable attention in the mathematical physics literature during the last twenty years, and a number of different approaches, based on scattering theory techniques, have been developed. In ref. 9 a kind of Floquet theory is used to analyze the case of time-dependent potentials decaying like $L^{1}$ functions in time. The approach has also been used to treat some problems involving time-periodic potentials and long range potentials. ${ }^{(17,43,10)}$

Another type of time-dependent problem is the charge-transfer problems. These are problems in which the potential $W$ is a sum of terms of the type: $W(x-v t)$. In ref. 45 a charge transfer problem is mapped in a similar way onto a three-body type problem which is treated by resolvent methods; In ref. 6 a geometric approach to scattering was used to study the general behavior of scattering states with time-dependent potentials.

For potentials decaying slowly in time which are perturbations of many body hamiltonians, which appear in the analysis of $\mathrm{N}$-body long range scattering, phase-space methods were used in ref. 35, based on the existence of an asymptotic energy operator in such cases. The AC Stark effect was treated in ref. 44 by methods exploiting the special form of the potential. Also related to our study is research concerning the wave equation with a time periodic potential or the free wave equation in the exterior of a periodically oscillating obstacle; see, for example, refs. 4 and 40 , and those cited therein.

We consider a Hamiltonian, $H_{0}$, with one bound state, perturbed by a small and localized potential, time-periodic potential of the simple form given in Hypothesis (H5). This assumption is made to simplify computations but the general method applies to problems with more than one 
bound state and to more general time-periodic or quasi-periodic perturbations. An application, currently under investigation involves the structural instability and meta-stability of "breather-like" modes of certain linear wave-guide problems related to "M-soliton" solutions of integrable nonlinear flows. ${ }^{(21)}$

In this paper we treat a model of the ionization problem. ${ }^{(19,7)}$ By treating the process of ionization as a resonance caused by coupling of the point spectrum to the continuous spectrum, we apply the time dependent resonance theory, recently developed in refs. 37 and 38 to find the large time behavior of the solution. In particular, we show that under our conditions, the bound state is always disintegrating and find the lifetime, $\tau \sim \Gamma^{-1}$, and the spectral line shift, (4.21); see the remark following Theorem 2.2. We also prove local decay estimates for the solution, which are of general interest to nonlinear and other problems.

\subsection{Notations and Terminology}

Throughout this paper we will denote a generic constant by $C, D$, etc. $\langle x\rangle=\left(1+|x|^{2}\right)^{1 / 2}$.

$\mathscr{L}(\mathscr{H})=$ the space of bounded linear operators on $\mathscr{H}$

Functions of self-adjoint operators are defined via the spectral theorem. ${ }^{(28)}$

\section{GENERAL FORMULATION AND MAIN RESULTS}

Consider the general system

$$
i \partial_{t} \phi(t)=\left(H_{0}+W(t)\right) \phi(t)
$$

Here, $\phi(t)$ denotes a function of time, $t$, with values in a complex Hilbert space $\mathscr{H}$.

Hypotheses on $H_{0}$

(H1) $H_{0}$ is self-adjoint on $\mathscr{H}$ and both $H_{0}$ and $W(t), t \in \mathbb{R}^{1}$, are densely defined on a subspace $\mathscr{D}$ of $\mathscr{H}$.

The norm on $\mathscr{H}$ is denoted by $\|\cdot\|$, and the inner product of $f, g \in \mathscr{H}$, by $(f, g)$.

(H2) The spectrum of $H_{0}$ is assumed to consist of an absolutely continuous part, $\sigma_{\text {cont }}\left(H_{0}\right)$, with associated spectral projection $\mathbf{P}_{\mathbf{c}}$ and a single isolated eigenvalue $\lambda_{0}$ with corresponding normalized eigenstate, $\psi_{0}$, i.e.,

$$
H_{0} \psi_{0}=\lambda_{0} \psi_{0}, \quad\left\|\psi_{0}\right\|=1
$$


The manner in which we shall measure the decay of solutions is typically in a local decay sense, e.g., for the scalar Schrödinger equation we measure local decay using the norms: $f \mapsto\left\|\langle x\rangle^{-\sigma} f\right\|_{L^{2}}$, where $\sigma>0$. So that our theory applies to a class of general systems (involving, for example, vector equations with matrix operators), we assume the existence of self-adjoint "weights," $w_{-}$and $w_{+}$such that $c>0$.

(i) $w_{+}$is defined on a dense subspace of $\mathscr{H}$ and on which $w_{+} \geqslant c I$,

(ii) $w_{-} \in \mathscr{L}(\mathscr{H})$

(iii) $w_{-} w_{+} \mathbf{P}_{\mathbf{c}}=\mathbf{P}_{\mathbf{c}}=\mathbf{P}_{\mathbf{c}} w_{-} w_{+}$

In the scalar case $w_{+}$and $w_{-}$correspond to multiplication by $\langle x\rangle^{\sigma}$ and $\langle x\rangle^{-\sigma}$, respectively; see Section 3.

The following hypothesis and Corollary ensure that the equation satisfies sufficiently strong dispersive time-decay estimates.

(H3) Local decay estimates on $e^{-i H_{0} t}$ : Let $r \geqslant 2+\varepsilon$, where $\varepsilon>0$. There exist $w_{+}$and $w_{-}$, as above, such that for all $f$ :

$$
\text { (a) }\left\|w_{-} e^{-i H_{0} t} \mathbf{P}_{\mathbf{c}} f\right\| \leqslant C\langle t\rangle^{-r+1}\left\|w_{+} f\right\|
$$

and

(b) $\left\|w_{-} e^{-i H_{0} t}\left(H_{0}-\lambda_{0}-\mu-i 0\right)^{-1} \mathbf{P}_{\mathbf{c}} f\right\| \leqslant C\langle t\rangle^{-r+1}\left\|w_{+} f\right\|$

(1) Typically one is not "handed" the singular estimate (2.4). It can however be proved using (2.3) and the following:

$\left(\mathbf{b}_{A}\right)$ Let $\Delta$ denote an open interval containing $\lambda_{0}+\mu$ and contained in the interior of the continuous spectrum of $H_{0}$. Let $\mathbf{g}_{\mathbf{\Delta}}$ denote a smooth function of compact support which is identically one on $\Delta$. Then, for all $f$ such that $w_{+} f \in \mathscr{H}$

$$
\left\|w_{-} e^{-i H_{0} t} \mathbf{g}_{\Delta} f\right\| \leqslant C_{\Delta}\langle t\rangle^{-\boldsymbol{r}}\left\|w_{+} f\right\|
$$

(2) To prove that (2.4) is implied by estimates (2.3) and (2.5), one can follow very closely the approach taken in refs. 37 and 38. In particular, see Proposition 2.1 and Appendix A of ref. 38. In ref. 38, the proofs are carried out for a particular choice of $\mathscr{H}, w_{+}$, and $w_{-}$, but as indicated in the remark following the main theorem of ref. 38, the proof can be adapted to a more general setting and is virtually unchanged; one need only replace $L^{2}$ by a general Hilbert space $\mathscr{H},\langle x\rangle^{-\sigma}$ by $w_{-}$, and $\langle x\rangle^{\sigma}$ by $w_{+}$. This requires hypothesis: 
(H4) There is a choice of real number $c$ such that

$$
\left\|w_{+}\left(H_{0}+c\right)^{-1} w_{-}\right\|_{\mathscr{L}(\mathscr{H})}
$$

can be made sufficiently small.

(H5) Hypotheses on the perturbation $W(t)$. We consider time-dependent symmetric perturbations of the form

$$
W(t)=\cos \mu t \beta, \quad \text { with } \quad \beta^{*}=\beta
$$

In many applications, $\beta$, is a spatially localized scalar or matrix function.

To measure the size of the perturbation $W$, we introduce the norm

$$
\|W\| \equiv\left(\left\|w_{+} \beta\right\|_{\mathscr{L}(\mathscr{H})}^{2}+\left(\left\|w_{+} \beta w_{+}\right\|_{\mathscr{L}(\mathscr{H})}^{2}\right)^{1 / 2}\right.
$$

Our final hypothesis is a resonance condition which says that $\mu+\lambda_{0} \in \sigma_{\text {cont }}\left(H_{0}\right)$ and that there is nontrivial coupling.

(H6) Resonance condition. There exists $\theta_{0}>0$, independent of $W$, such that

$$
\Gamma \equiv \frac{\pi}{4}\left(\beta \psi_{0}, \delta\left(H_{0}-\lambda_{0}-\mu\right) \beta \psi_{0}\right) \geqslant \theta_{0}\|W\|^{2}>0
$$

Remark. That the expression in (2.9) and the principal value term in (2.13) below are finite is a consequence ${ }^{(2)}$ of the local decay estimate (H3) and the representation:

$$
\left(\beta \psi_{0},\left(H_{0}-\lambda-\mu-i 0\right)^{-1} \beta \psi_{0}\right)=i \int_{0}^{\infty}\left(\beta \psi_{0}, e^{-i\left(H_{0}-\lambda_{0}-\mu\right) s} \beta \psi_{0}\right) d s
$$

Our main result is the following:

Theorem 2.1. Let $H_{0}$ satisfy hypotheses (H1)-(H6) and assume $w_{+} \phi_{0} \in \mathscr{H}$. Then, if $\|W\|$ is sufficiently small, the solution of (2.1) satisfies the local decay estimate:

$$
\left\|w_{-} \phi(t)\right\| \leqslant C\langle t\rangle^{-r+1}\left\|w_{+} \phi_{0}\right\|, \quad t \in \mathbb{R}
$$


Remarks. In the proof we actually use a less stringent property than (H6). Rather, we use that:

(H6') $\|W\|^{3} \Gamma^{-1}$ is sufficiently small.

Under the same hypotheses as Theorem 2.1, we obtain more detailed information on the behavior of $\phi(t)$ :

\section{Theorem 2.2.}

$$
\begin{aligned}
\phi(x, t) & =a(t) \psi_{0}+\phi_{d}(t), \quad\left(\psi_{0}, \phi_{d}(t)\right)=0 \\
a(t) & =a(0) e^{-\Gamma t} e^{-i \omega_{\mathbf{l}}(t)}+R_{a}(t) \\
\phi_{d}(t) & =e^{-i H_{0} t} \mathbf{P}_{\mathbf{c}} \phi_{0}+\tilde{\phi}(t)
\end{aligned}
$$

For any fixed $T_{0}>0$, we have

$$
\left|R_{a}(t)\right| \leqslant C\|W\|, \quad|t| \leqslant T_{0} \Gamma^{-1}
$$

Moreover,

$$
\begin{aligned}
\left|R_{a}(t)\right|= & \mathscr{U}\left(\langle t\rangle^{-r+1}\right), \quad|t| \rightarrow \infty \\
\omega_{1}(t)= & \lambda_{0} t-\frac{1}{4}\left(\beta \psi_{0},\left(H_{0}-\lambda_{0}+\mu\right)^{-1} \mathbf{P}_{\mathbf{c}} \beta \psi_{0}\right) t \\
& -\frac{1}{4}\left(\beta \psi_{0}, \text { P.V. }\left(H_{0}-\lambda_{0}-\mu\right)^{-1} \mathbf{P}_{\mathbf{c}} \beta \psi_{0}\right) t+M(t)
\end{aligned}
$$

where $M(t)$ is of order $\|W\|^{2}$ and is oscillatory in $t$. Furthermore, $\tilde{\phi}=$ $\phi_{1}+\phi_{2}$ is explicitly given in (4.9), with $\left\|w_{-} \tilde{\phi}(t)\right\|=\left(\cdot\left(\langle t\rangle^{-r+1}\right)\right.$ as $|t| \rightarrow \infty$, and therefore, by (H3) same holds $\left\|w_{-} \phi_{d}(t)\right\|=c\left(\langle t\rangle^{-r+1}\right)$ as $|t| \rightarrow \infty$.

Remark. Suppose the initial data is given by the bound state of the unperturbed problem, i.e., $\phi(x, 0)=\psi_{0}(x), a(0)=1, \phi_{d}(0)=0$. Then, from the expansion of the solution we have that for $0 \leqslant t \leqslant \Gamma^{-1}$ that the projection of the solution on $\psi_{0}$ is of order $e^{-\Gamma t}$, with an error of order $\|W\|$. Hence it is natural to view the state $\psi_{0} e^{-i \lambda_{0} t}$ as a metastable state, with lifetime $\tau=\Gamma^{-1} \sim\|W\|^{-2}$.

\section{AN EXAMPLE: THE SCHRÖDINGER EQUATION}

In this section we apply the results of Theorems 2.1 and 2.2 to the special case of the Schrödinger equation with a time-periodic and spatially localized perturbing potential, Eq. (1.3). Models of the sort considered in this example occur in the study of ionization of an atom by a time-varying electric field; see refs. 19 and 7. 
We begin by indicating assumptions on $V(x)$ and $\beta(x)$ under which (H1)-(H6) hold.

We take $\mathscr{H}=L^{2}\left(\mathbb{R}^{n}\right)$, and $H_{0} \equiv-\Delta+V(x)$, where $V(x)$ and $\beta(x)$ are real-valued, sufficiently differentiable and decaying rapidly as $x \in \mathbb{R}^{n}$ tends to infinity. Thus, $H_{0}$ is self-adjoint, and $H_{0}$ and $W(t)$ are densely defined operators in $L^{2}$. Moreover, we assume that $H_{0}$ has exactly one negative eigenvalue and the absolutely continuous spectrum of $H_{0}$ is equal to the positive half-line.

With regard hypothesis $(\mathrm{H} 3)$, we take the weights used to measure local energy decay to be $w_{ \pm} \equiv\langle x\rangle^{ \pm \sigma}$. There is a good deal of literature on local energy decay estimates for $e^{-i H_{0} t} \mathbf{P}_{\mathrm{c}}$. These results require sufficient regularity and decay of the potential $V(x)$. We refer the reader to refs. 13, 15 and 23; see also refs. 27 and 31.

The large time expansion of the operator: $\langle x\rangle^{-\sigma} e^{-i H_{0} t} \mathbf{P}_{\mathbf{c}}\langle x\rangle^{-\sigma}$ in $L^{2}$ contains a non-generic leading order term of order $t^{-1 / 2}$. More rapid decay as $t \rightarrow \infty$ is ensured provided we assume the following condition concerning the behavior of the resolvent of $H_{0}$ near zero energy:

(Z) Zero is not a resonance of the operator $-A+V(x)$. For a precise definition of zero energy resonance in this context and a detailed discussion see refs. 13 and 23 . tials.

Remark. The condition ( $\mathrm{Z}$ ) holds generically in the space of poten-

Hypothesis $(\mathrm{H} 3 \mathrm{a})$ is a consequence of:

Proposition 3.1. Assume that $V(x)$ is sufficiently regular and decays sufficiently rapidly as $|x| \rightarrow \infty$; see refs. 13,15 , and 23 . Additionally, assume condition $(Z)$. Then, we have the following local energy decay estimate.

(a) Assume $n \geqslant 3$ and $\sigma>1$. Then, there exists $r>2$ such that

$$
\left\|\langle x\rangle^{-\sigma} e^{-i H_{0} t} \mathbf{P}_{\mathbf{c}} f\right\|_{2} \leqslant C\langle t\rangle^{-r+1}\left\|\langle x\rangle^{\sigma} f\right\|_{2}
$$

(b) Assume $n=1$. Then, we get (3.1) with $\langle t\rangle^{-r+1}=\langle t\rangle^{-3 / 2}$.

(c) Assume $n=2$. Then, we get (3.1) with $\langle t\rangle^{-r+1}$ replaced by $\left\langle t \log ^{2} t\right\rangle^{-1}$.

Hypothesis $\left(\mathbf{H} 3 \mathbf{b}_{A}\right)$ is a consequence of:

Proposition 3.2. Assume the above hypotheses on $H_{0}$ and assume that $\Delta$ denotes an open and bounded subinterval of the continuous spectrum 
of $H_{0}$. Let $g_{A}$ denote a smooth characteristic function, as defined in Section 2. Then, for $2<r<\sigma$,

$$
\left\|\langle x\rangle^{-\sigma} e^{-i H_{0} t} g_{\Delta}\left(H_{0}\right) f\right\|_{2} \leqslant C\langle t\rangle^{-r}\left\|\langle x\rangle^{\sigma} f\right\|_{2}
$$

Proof. If $H_{0}$ is replaced by the Laplacian, this estimate is straightforward since $g_{\Delta}\left(H_{0}\right) f$ is spectrally supported away from points of stationary phase. An approach to this estimate for $H_{0}$, a variable coefficient operator is reviewed in Appendix A and is based on the Mourre estimate, where the operator $A$ can be taken to be: $A=(x \cdot p+p \cdot x) / 2$, where $p=-i \nabla$.

We take the time-dependent perturbation to be of the form:

$$
W(x, t)=\cos \mu t \beta(x)
$$

with $\beta$ sufficiently differentiable and rapidly decaying in $x$, e.g., $\langle x\rangle^{2 \sigma} \beta(x)$ $\in L^{2}$. Thus, (H5) is satisfied as well. Finally, the resonance condition (H6), holds generically in the potential $V(x)$.

Therefore, our main theorems Theorems 2.1 and Theorems 2.2 on the structural instability of the unperturbed bound state, and large time behavior of such systems apply.

\section{DECOMPOSITION AND DERIVATION OF THE DISPERSIVE NORMAL FORM}

As in refs. 37 and 38, we begin by deriving a decomposition of the solution, $\phi(t)$, which will facilitate the study of its large time behavior. Let

$$
\phi(t)=a(t) \psi_{0}+\phi_{d}(t)
$$

with the orthogonality condition

$$
\left(\psi_{0}, \phi_{d}\right)=0 \quad \text { for all } t
$$

Note therefore that $\phi_{d}=\mathbf{P}_{\mathrm{c}} \phi_{d}$ since $H_{0}$ has been assumed to have only one eigenvalue.

Remark. The required modifications in our analysis for the case where $W(t)$ has more general periodic or quasi-periodic time dependence, and when $H_{0}$ has more than one bound state can be seen at this point. If $H_{0}$ has $M$ discrete eigenvalues, then the decomposition (4.1) is replaced by a sum over the $M$ discrete modes with amplitudes $a_{j}(t), j=0, \ldots, M-1$, plus the continuous spectral part. If $W(t)$ has more general periodic or quasiperiodic time dependence, in the analysis of the coupled equations for $a_{j}(t)$ 
and $\phi_{d}(t)$ (see below), we use an expansion of $W(t)$ in terms of operators of the form (2.7).

We proceed by first inserting (4.1) into (2.1), which yields the equation:

$$
\begin{aligned}
i \partial_{t} a(t) & \psi_{0}+i \partial_{t} \phi_{d}(t) \\
= & \lambda_{0} a(t) \psi_{0}+H_{0} \phi_{d}(t)+a(t) W(t) \psi_{0}+W(t) \phi_{d}(t)
\end{aligned}
$$

Taking the inner product of (4.3) with $\psi_{0}$ yields the following equation for $a(t)$ :

$$
\begin{aligned}
& i \partial_{t} a=\lambda_{0} a(t)+\left(\psi_{0}, W(t) \psi_{0}\right) a(t)+\left(\psi_{0}, W(t) \phi_{d}\right) \\
& a(0)=\left(\psi_{0}, \phi(0)\right)
\end{aligned}
$$

In deriving (4.4) we have used that $\psi_{0}$ is normalized and the relation

$$
\left(\psi_{0}, \partial_{t} \phi_{d}\right)=0
$$

a consequence of $(4.2)$.

Applying $\mathbf{P}_{\mathbf{c}}$ to (4.3), we obtain an equation for $\phi_{d}$ :

$$
\begin{aligned}
i \hat{C}_{t} \phi_{d}(t) & =H_{0} \phi_{d}(t)+\mathbf{P}_{\mathbf{c}} W(t) \phi_{d}(t)+a(t) \mathbf{P}_{\mathbf{c}} W(t) \psi_{0} \\
\phi_{d}(0) & =\mathbf{P}_{\mathbf{c}} \phi(0)
\end{aligned}
$$

Since we are after a slow resonant decay phenomenon, it will prove advantageous to extract the fast oscillatory behavior of $a(t)$. We therefore define:

$$
A(t) \equiv e^{i \lambda_{0} t} a(t)
$$

Then, (4.4) reads

$$
\partial_{t} A=-i A\left(\psi_{0}, W(t) \psi_{0}\right)-i e^{i \lambda_{0} t}\left(\psi_{0}, W(t) \phi_{d}(t)\right)
$$

Solving (4.6) by Duhamel's formula we have

$$
\begin{aligned}
\phi_{d}(t)= & e^{-i H_{0} t} \phi_{d}(0)-i \int_{0}^{t} e^{-i H_{0}(t-s)} \mathbf{P}_{\mathbf{c}} W(s) a(s) \psi_{0} d s \\
& -i \int_{0}^{t} e^{-i H_{0}(t-s)} \mathbf{P}_{\mathbf{c}} W(s) \phi_{d}(s) d s \\
\equiv & \phi_{0}(t)+\phi_{1}(t)+\phi_{2}(t)
\end{aligned}
$$


By standard methods, the system (4.8)-(4.9) for $A(t)$ and $\phi_{d}(t)=$ $\phi(t)-e^{-i \lambda_{0} t} A(t) \psi_{0}$ has a global solution in $t$ with

$$
A \in C^{1}(\mathbb{R}), \quad\left\|\phi_{d}(t)\right\| \in C^{0}(\mathbb{R}), \quad\left\|w_{-} \phi_{d}(t)\right\| \in C^{0}(\mathbb{R})
$$

Our analysis of the $|t| \rightarrow \infty$ behaviour is based on a study of this system.

We next insert (4.9) into (4.8) we get

$$
\partial_{t} A(t)=-i A(t)\left(\psi_{0}, W(t) \psi_{0}\right)-i e^{i \lambda_{0} t} \sum_{j=0}^{2}\left(\psi_{0}, W(t) \phi_{j}\right)
$$

We next give a detailed expansion of the sum in (4.10). It is in the $j=1$ term that the key resonance is found. This makes it possible to find a normal form for (4.10) in which internal damping in the system is made explicit. This damping is responsible for the energy transfer from the discrete to continuum modes of the system and the associated radiative decay of solutions.

\section{Proposition 4.1.}

$$
\begin{aligned}
-i e^{i \lambda_{0} t} \cos \mu t\left(\beta \psi_{0}, \phi_{1}(t)\right)=R_{1}+R_{2}+R_{3}+R_{4}, \quad \text { where } \\
R_{1}=-\frac{1}{2} e^{i \lambda_{0} t} \cos \mu t \int_{0}^{t}\left(\beta \psi_{0}, e^{-i H_{0}(t-s)} e^{i\left(\mu-\lambda_{0}\right) s} A(s) \mathbf{P}_{\mathbf{c}} \beta \psi_{0}\right) d s \\
R_{2}=\frac{i}{2} e^{-i \mu t} \cos \mu t A(t)\left(\beta \psi_{0},\left(H_{0}-\lambda_{0}-\mu-i 0\right)^{-1} \mathbf{P}_{\mathbf{c}} \beta \psi_{0}\right) \\
R_{3}=-\frac{i}{2} A(0) e^{i \lambda_{0} t} \cos \mu t\left(\beta \psi_{0}, e^{-i H_{0} t}\left(H_{0}-\lambda_{0}-\mu-i 0\right)^{-1} \mathbf{P}_{\mathbf{c}} \beta \psi_{0}\right) \\
R_{4}=-\frac{i}{2} e^{i \lambda_{0} t} \cos \mu t \int_{0}^{t}\left(\beta \psi_{0}, e^{-i H_{0}(t-s)}\left(H_{0}-\lambda_{0}-\mu-i 0\right)^{-1}\right. \\
\left.\quad \times e^{-i\left(\lambda_{0}+\mu\right) s} \partial_{s} A(s) \mathbf{P}_{\mathbf{c}} \beta \psi_{0}\right) d s
\end{aligned}
$$

Proof of Proposition 4.1. Using that $\cos \mu t=\frac{1}{2}\left(e^{i \mu t}+e^{-i \mu t}\right)$ and the definition $A(t)=e^{i \lambda_{0} t} a(t)$, we get from (4.9)

$$
\begin{aligned}
\phi_{1}(t)= & -\frac{i}{2} \int_{0}^{t} e^{-i H_{0}(t-s)} e^{-i\left(\lambda_{0}-\mu\right) s} A(s) \mathbf{P}_{\mathbf{c}} \beta \psi_{0} d s \\
& -\frac{i}{2} \int_{0}^{t} e^{-i H_{0}(t-s)} e^{-i\left(\lambda_{0}+\mu\right) s} A(s) \mathbf{P}_{\mathbf{c}} \beta \psi_{0} d s \\
\equiv & K_{0}+K_{\mathrm{res}}
\end{aligned}
$$


Therefore,

$$
\begin{aligned}
-i e^{i \lambda_{0} t} \cos \mu t\left(\beta \psi_{0}, \phi_{1}(t)\right) & =-i e^{i \lambda_{0} t} \cos \mu t\left(\beta \psi_{0}, K_{0}+K_{\mathrm{res}}\right) \\
& =R_{1}-i e^{i \lambda_{0} t} \cos \mu t\left(\beta \psi_{0}, K_{\mathrm{res}}\right)
\end{aligned}
$$

The terms $R_{j}, j=2,3,4$ in (4.11) come from an expansion of the last term in (4.13). This is the source of the key resonant contribution. We now proceed with a careful evaluation of this term.

We first regularize $K_{\text {res }}$. For $t>0$, let $\varepsilon$ be positive and arbitrary and define:

$$
K_{\mathrm{res}}^{\varepsilon}=-\frac{i}{2} \int_{0}^{t} e^{-i H_{0}(t-s)} e^{-i\left(\lambda_{0}+\mu-i \varepsilon\right) s} A(s) \mathbf{P}_{\mathrm{c}} \beta \psi_{0} d s
$$

Note that $K_{\text {res }}=\lim _{\varepsilon \rightarrow 0} K_{\text {res }}^{\varepsilon}$. Integration of the expression in (4.14) by parts, and letting $\varepsilon$ tend to zero from above gives

Proposition 4.2. The following expansion of $K_{\text {res }}$ is valid in $\mathscr{P}^{\prime}$ :

$$
\begin{aligned}
K_{\text {res }}= & -\frac{1}{2} e^{-i\left(\lambda_{0}+\mu\right) t} A(t)\left(H_{0}-\lambda-\mu-i 0\right)^{-1} \mathbf{P}_{\mathbf{c}} \beta \psi_{0} \\
& +\frac{1}{2} A(0) e^{-i H_{0} t}\left(H_{0}-\lambda-\mu-i 0\right)^{-1} \mathbf{P}_{\mathbf{c}} \beta \psi_{0} \\
& +\frac{1}{2} \int_{0}^{t} e^{-i H_{0}(t-s)}\left(H_{0}-\lambda-\mu-i 0\right)^{-1} e^{-i\left(\lambda_{0}+\mu\right) s} \partial_{s} A(s) \mathbf{P}_{\mathbf{c}} \beta \psi_{0} d s
\end{aligned}
$$

The choice of regularization, $-i \varepsilon$, in (4.14) ensures that the latter two terms in the expansion of $K_{\text {res }},(4.15)$, decay dispersively as $t \rightarrow+\infty$; see hypothesis (H3) and Section 6. For $t<0$, we replace $+i \varepsilon$ with $-i \varepsilon$ in (4.14).

The proof of Proposition 4.1 is now completed by substitution of the expansion (4.15) for $K_{\text {res }}$ into the last term in (4.13).

We further expand the terms $R_{1}$ and $R_{2}$ to make explicit the resonant contributions.

Proposition 4.3.

$$
\begin{aligned}
& R_{1}=\rho_{1}(t) A(t)+R_{1 a}(t)+R_{1 b}(t) \\
& R_{2}=\left(\rho_{2}(t)-\Gamma\right) A(t)
\end{aligned}
$$


where

$$
\begin{aligned}
\rho_{1}(t)= & \frac{i}{4}\left(1+e^{2 i \mu t}\right)\left(\beta \psi_{0},\left(H_{0}-\lambda_{0}+\mu\right)^{-1} \mathbf{P}_{\mathbf{c}} \beta \psi_{0}\right) \\
\rho_{2}(t)= & \frac{i}{4}\left(1+e^{-2 i \mu t}\right)\left(\beta \psi_{0}, \text { P.V. }\left(H_{0}-\lambda_{0}-\mu\right)^{-1} \mathbf{P}_{\mathbf{c}} \beta \psi_{0}\right) \\
& -\frac{\pi}{4} e^{-2 i \mu t}\left(\beta \psi_{0}, \delta\left(H_{0}-\lambda_{0}-\mu\right) \mathbf{P}_{\mathbf{c}} \beta \psi_{0}\right) \\
\Gamma= & \frac{\pi}{4}\left(\beta \psi_{0}, \delta\left(H_{0}-\lambda_{0}-\mu\right) \mathbf{P}_{\mathbf{c}} \beta \psi_{0}\right) \\
R_{1 a}(t)= & -\frac{i}{2} A(0) e^{i \lambda_{0} t} \cos \mu t\left(\beta \psi_{0}, e^{-i H_{0} t}\left(H_{0}-\lambda_{0}+\mu\right)^{-1} \mathbf{P}_{\mathbf{c}} \beta \psi_{0}\right) \\
R_{1 b}(t)= & \frac{1}{2 i} e^{i \lambda_{0} t} \cos \mu t\left(\beta \psi_{0}, \int_{0}^{t} e^{-i H_{0}(t-s)} e^{-i\left(\lambda_{0}-\mu\right) s} \partial_{s} A(s)\right. \\
& \left.\times\left(H_{0}-\lambda+\mu\right)^{-1} \mathbf{P}_{\mathrm{c}} \beta \psi_{0}\right)
\end{aligned}
$$

Proof. To obtain (4.17) we use the well known distributional identity

$$
(x-i 0)^{-1}=\text { P.V. } x^{-1}+i \pi \delta(x)
$$

Equation (4.16) follows by integration by parts.

Combining Eq. (4.10) with the previous propositions, we have

\section{Proposition 4.4.}

$$
\partial_{t} A(t)=(-\Gamma+\rho(t)) A(t)+E(t)
$$

where

$$
\begin{aligned}
E(t)= & R_{1 a}+R_{1 b}+R_{3}+R_{4}-i e^{i \lambda_{0} t} \cos \mu t\left(\beta \psi_{0}, \phi_{0}(t)\right) \\
& -i e^{i \lambda_{0} t} \cos \mu t\left(\beta \psi_{0}, \phi_{2}\right)
\end{aligned}
$$


Here, $\phi_{0}$ and $\phi_{2}$ are given in (4.9), $R_{1 a}$ and $R_{1 b}$ in (4.18), $R_{3}$ and $R_{4}$ in (4.11), and

$$
\begin{aligned}
\rho(t) \equiv & -i \cos \mu t\left(\psi_{0}, \beta \psi_{0}\right)+\rho_{1}(t)+\rho_{2}(t) \\
= & \frac{i}{4}\left(\beta \psi_{0},\left(H_{0}-\lambda_{0}+\mu\right)^{-1} \mathbf{P}_{\mathbf{c}} \beta \psi_{0}\right) \\
& +\frac{i}{4}\left(\beta \psi_{0}, \text { P.V. }\left(H_{0}-\lambda_{0}-\mu\right)^{-1} \mathbf{P}_{\mathbf{c}} \beta \psi_{0}\right)+m(t)
\end{aligned}
$$

where $m(t)$ is a bounded and oscillatory function of zero mean.

Remarks. (1) The point of (4.19) is that the damping coefficient $(\Gamma>0)$, which arises due to the coupling of the discrete bound state to the continuum modes by the periodic perturbation, is made explicit. This facilitates a direct study of the transfer of energy from the bound state to the continuum and the associated radiative decay of solutions.

(2) The leading order part of Eq. (4.19) is the analogue of the dispersive normal form derived in ref. 39 for a class of nonlinear dispersive wave equations.

In the next section we estimate the remainder terms in (4.9) and (4.19).

\section{ODE ESTIMATES}

Equations (4.9) and (4.19) comprise a dynamical system governing $\phi_{d}(t)$ and $a(t)$, the solution of which is equivalent to the original Eq. (1.1). Our aim in this decomposition is to separate the dispersive (polynomially time-decaying) part $\phi_{d}(t)$ from the solution, as well as the transient (exponentially time-decaying) part. In this and in the following section we derive a coupled system of estimates for $A(t)$ and $\phi_{d}(t)$. We show that $A(t)$ decays in time, provided $\phi_{d}(t)$ is dispersively decaying and vice-versa. Then, we exploit the assumption that the potential, $W$, is small to close the resulting inequalities, and prove the decay of both $A(t)$ and $\phi_{d}(t)$.

At this point, we state a simple Lemma which we shall use in a number of places in this and in the next section.

Lemma 5.1. Let $\alpha>1$.

$$
\int_{0}^{t}\langle t-s\rangle^{-\alpha}\langle s\rangle^{-\beta} d s \leqslant C_{\alpha, \beta}\langle t\rangle^{-\min (\beta, \beta+\alpha-1)}
$$


Proof. The bound is obtained by viewing the integral as decomposed into a part over $[0, t / 2]$ and the part over $[t / 2, t]$. We estimate the integral over $[0, t / 2]$ by bounding $\langle t-s\rangle^{-\alpha}$ by its value at $t / 2$ and explicitly computing the remaining integral. The integral over $[t / 2, t]$ is computed by bounding $\langle s\rangle^{-\beta}$ by its value at $t / 2$ and again computing explicitly the remaining integral. Putting the two estimates together yields the lemma.

We now turn to the estimate for $A(t)$ in terms of the dispersive norm of $\phi_{d}(t)$ and local decay estimates for $e^{-i H_{0} t} \mathbf{P}_{\mathbf{c}}\left(H_{0}\right)$.

Proposition 5.1. $A(t)$, the solution of (4.19), can be expanded as:

$$
\begin{aligned}
A(t) & =e^{\int_{0}^{t} \rho(s) d s}\left(e^{-\Gamma t} A(0)+R_{A}(t)\right) \\
R_{A}(t) & =\int_{0}^{t} e^{-\Gamma(t-\tau)} \tilde{E}(\tau) d \tau
\end{aligned}
$$

where $\widetilde{E}(t)$ is given in (4.20) and (5.9). For any $\alpha>1, R_{A}(t)$ satisfies the estimates:

$$
\begin{aligned}
& \sup _{2 \Gamma^{-\alpha} \leqslant t \leqslant T}\langle t\rangle^{r-1}\left|R_{A}(t)\right| \leqslant C_{1} e^{-\Gamma^{-\delta}} \sup _{0 \leqslant \tau \leqslant \Gamma^{-\alpha}}|E(\tau)| \\
&+C \Gamma^{-1} \sup _{\Gamma^{-\alpha} \leqslant \tau \leqslant T}\left(\langle\tau\rangle^{r-1}|E(\tau)|\right) \\
& \sup _{0 \leqslant t \leqslant 2 \Gamma^{-\alpha}}\langle t\rangle^{r-1}\left|R_{A}(t)\right| \leqslant D \Gamma^{-\alpha r} \sup _{0 \leqslant \tau \leqslant 2 \Gamma^{-\alpha}}|E(\tau)|
\end{aligned}
$$

Proof. To prove (5.4) we begin with (4.19)

$$
\partial_{t} A(t)=\rho(t) A(t)-\Gamma A(t)+E(t)
$$

where $E(t)$ is given by $(4.20)$. Let

$$
\tilde{A}(t) \equiv e^{-\int_{0}^{t} \rho(s) d s} A(t)
$$

Then, $\tilde{A}$ satisfies the equation

$$
\begin{aligned}
& \partial_{t} \tilde{A}=-\Gamma \tilde{A}+\tilde{E}(t) \\
& \tilde{E}(t)=e^{-\int_{0}^{t} \rho(s) d s} E(t)
\end{aligned}
$$

Solving (5.8) we get

$$
\begin{aligned}
\tilde{A}(t) & =e^{-\Gamma t} \tilde{A}(0)+\int_{0}^{t} e^{-\Gamma(t-s)} \widetilde{E}(s) d s \\
& \equiv e^{-\Gamma t} \tilde{A}(0)+R_{A}(t)
\end{aligned}
$$


Since the real part of $\rho(t)$ is of the general form of $c_{1} \cos \omega_{1} t+$ $c_{2} \sin \omega_{2} t$, its integral is bounded uniformly in $t$. Therefore,

$$
C^{-1}|\tilde{A}(t)| \leqslant|A(t)| \leqslant C|\tilde{A}(t)| \quad \text { for some } \quad C>0
$$

and similarly for $E(t), \widetilde{E}(t)$. It is therefore sufficient to estimate $\tilde{A}(t)$, in terms of $\tilde{E}(t)$.

Remark. Estimates of $R_{a}(t)$, which appears in the statement of Theorem 2.2, are related to those for $R_{A}(t)$ via:

$$
\left|R_{a}(t)\right|=\left|e^{-f_{0}^{t} \rho(s) d s} R_{A}(t)\right| \leqslant C\left|R_{A}(t)\right|
$$

Note that on any fixed time interval, $0 \leqslant t \leqslant 2 M$, one has by Gronwall type estimates that, $|\tilde{A}(t)|$ and $\left\|w_{-} \phi_{d}(t)\right\|$ are bounded by a constant depending on the initial data and $M$. This bound is, in general, exponentially large in $M$. We shall now focus on obtaining appropriate bounds for these quantities on $t$-intervals, $[2 M, T)$, which are independent of $T$.

From (5.10) we have for any $M>0$ :

$$
\begin{aligned}
|\widetilde{A}(t)| & \leqslant|A(0)| e^{-\Gamma t}+\int_{0}^{M} e^{-\Gamma(t-s)}|\tilde{E}(s)| d s+\int_{M}^{t} e^{-\Gamma(t-s)}|\tilde{E}(s)| d s \\
& =|A(0)| e^{-\Gamma t}+I_{1}(t)+I_{2}(t)
\end{aligned}
$$

Set

$$
M=\Gamma^{-\alpha}, \quad \alpha>1
$$

We now estimate the terms $I_{1}(t)$ and $I_{2}(t)$ in (5.13) for $2 T^{-\alpha} \leqslant t \leqslant T$.

$$
\begin{aligned}
\langle t\rangle^{r-1} I_{1}(t) & =\langle t\rangle^{r-1} \int_{0}^{M} e^{-\Gamma(t-s)}|\widetilde{E}(s)| d s \\
& \leqslant\langle t\rangle^{r-1} e^{-1 / 2 \Gamma t} \cdot \int_{0}^{M} e^{-\Gamma(1 / 2 t-s)} d s \cdot \sup _{0 \leqslant \tau \leqslant \Gamma^{-\alpha}}|\tilde{E}(\tau)| \\
& \leqslant \sup _{2 \Gamma^{-\alpha} \leqslant \tau \leqslant T}\left(\langle t\rangle^{r-1} e^{-\Gamma t / 2}\right) \cdot C \Gamma^{-1} \cdot \sup _{0 \leqslant \tau \leqslant \Gamma^{-\alpha}}|\widetilde{E}(\tau)| \\
& \leqslant C e^{-\Gamma^{-\delta}} \sup _{0 \leqslant \tau \leqslant \Gamma^{-\alpha}}|\widetilde{E}(\tau)|
\end{aligned}
$$


for some $\delta>0$. Therefore,

$$
\sup _{2 \Gamma^{-\alpha} \leqslant \tau \leqslant T}\left(\langle t\rangle^{r-1} I_{1}(t)\right) \leqslant C e^{-\Gamma^{-\delta}} \sup _{0 \leqslant \tau \leqslant \Gamma^{-\alpha}}|\widetilde{E}(\tau)|
$$

We estimate $I_{2}(t)$ on the interval $2 \Gamma^{-\alpha} \leqslant t \leqslant T$ as follows:

$$
\langle t\rangle^{r-1} I_{2}(t) \leqslant\langle t\rangle^{r-1} \int_{\Gamma^{-\alpha}}^{t} e^{-\Gamma(t-s)}\langle s\rangle^{-r+1} d s \sup _{\Gamma^{-\alpha} \leqslant \tau \leqslant T}\left(\langle\tau\rangle^{r-1} \tilde{E}(\tau)\right)
$$

The integral is now bounded above using the estimate

$$
\langle t\rangle^{r-1} \int_{\Gamma^{-\alpha}}^{t} e^{-\Gamma(t-s)}\langle s\rangle^{-r+1} d s \leqslant C \Gamma^{-1}, \quad t \geqslant 2 \Gamma^{-\alpha}
$$

This gives

$$
\sup _{2 \Gamma^{-\alpha} \leqslant t \leqslant T}\langle t\rangle^{r-1} I_{2}(t) \leqslant C \Gamma^{-1} \sup _{\Gamma^{-\alpha} \leqslant \tau \leqslant T}\left(\langle\tau\rangle^{r-1} \tilde{E}(\tau)\right)
$$

Assembling the estimates (5.15) and (5.18) yields estimate (5.4) of Proposition 5.1. Estimate (5.5) is a simple consequence of the definition of $R_{A}(t)$.

\section{DISPERSIVE ESTIMATES AND LOCAL DECAY}

In this section we prove the local decay of $\phi_{d}$ and the decay in time of the remainder terms in the ODE of Section 4. To this end we will repeatedly use the following

Lemma 6.1. For any $\eta \in[0, r-1]$

$$
\left\|\int_{0}^{t} w_{-} e^{-i H_{0}(t-s)} \mathbf{P}_{\mathbf{c}} f(s) d s\right\| \leqslant C\langle t\rangle^{-\eta} \sup _{0 \leqslant \tau \leqslant t}\left(\langle\tau\rangle^{\eta}\left\|w_{+} f(\tau)\right\|\right)
$$

and

$$
\begin{aligned}
& \left\|\int_{0}^{t} w_{-} e^{-i H_{0}(t-s)} \mathbf{P}_{\mathbf{c}}\left(H_{0}-\lambda_{0}-\mu-i 0\right)^{-1} f(s) d s\right\| \\
& \leqslant C\langle t\rangle^{-\eta} \sup _{0 \leqslant \tau \leqslant t}\left(\langle\tau\rangle^{\eta}\left\|w_{+} f(\tau)\right\|\right)
\end{aligned}
$$


Proof. The proof follows from the assumed local decay estimates on $e^{-i H_{0} t}$; see (H3). Namely, using that $r>2$,

$$
\begin{aligned}
& \left\|\int_{0}^{t} w_{-} e^{-i H_{0}(t-s)} \mathbf{P}_{\mathbf{c}} f(s) d s\right\| \\
& \quad \leqslant \int_{0}^{t}\left\|w_{-} e^{-i H_{0}(t-s)} \mathbf{P}_{\mathbf{c}} w_{-}\right\|_{\mathscr{L}(\mathscr{H})}\langle s\rangle^{-\eta} d s \sup _{0 \leqslant \tau \leqslant t}\left(\langle\tau\rangle^{\eta}\left\|w_{+} f(\tau)\right\|\right) \\
& \quad \leqslant C \int_{0}^{t}\langle t-s\rangle^{-r+1}\langle s\rangle^{-\eta} d s \sup _{0 \leqslant \tau \leqslant t}\left(\langle\tau\rangle^{\eta}\left\|w_{+} f(\tau)\right\|\right) \\
& \quad \leqslant C\langle t\rangle^{-\eta} \sup _{0 \leqslant \tau \leqslant t}\left(\langle\tau\rangle^{\eta}\left\|w_{+} f(\tau)\right\|\right)
\end{aligned}
$$

which proves (6.1). The proof of (6.2) is identical, and uses the singular local decay estimate of $(\mathrm{H} 3)$.

We now define the norms

$$
[A]_{\alpha}(T)=\sup _{0 \leqslant \tau \leqslant T}\langle\tau\rangle^{\alpha}|A(\tau)|
$$

and

$$
\left[\phi_{d}\right]_{L D, \alpha}(T)=\sup _{0 \leqslant \tau \leqslant T}\langle\tau\rangle^{\alpha}\left\|w_{-} \phi_{d}(\tau)\right\|
$$

Then we have

Proposition 6.1. For any $T>0$,

$$
\left[\phi_{d}\right]_{L D, r-1}(T) \leqslant C\left(\left\|w_{+} \phi_{d}(0)\right\|+\|W\|[A]_{r-1}(T)\right)
$$

Proof. From Eq. (4.9) we get, using the assumed local decay estimate for $e^{-i H_{0} t}$ and (6.1),

$$
\begin{aligned}
\left\|w_{-} \phi_{d}(t)\right\| \leqslant & \sum_{j=0}^{2}\left\|w_{-} \phi_{j}(t)\right\| \\
\leqslant & C\langle t\rangle^{-r+1}\left\|w_{+} \phi_{d}(0)\right\|+C\langle t\rangle^{-r+1} \\
& \times[A]_{r-1}(t) \sup _{0 \leqslant s \leqslant t}\left\|w_{+} W(s) \psi_{0}\right\| \\
& +C\|W\|\langle t\rangle^{-r+1}\left[\phi_{d}\right]_{L D, r-1}(t)
\end{aligned}
$$


Since $\left\|w_{+} W(s) \psi_{0}\right\| \leqslant\|W\|\left\|\psi_{0}\right\|=\|W\|$ and $\|W\|$ is assumed to be small, multiplying both sides of this last equation by $\langle t\rangle^{r-1}$ and taking supremum over $t \leqslant T$ yields $(6.5)$.

We now estimate $E(t)$.

Proposition 6.2. Let $T>0$. For any $\eta \in[0, r-1]$ :

$$
[E]_{\eta}(T) \leqslant C\left(\|W\|^{2}|A(0)|+\|W\|\left\|w_{+} \phi_{d}(0)\right\|+\|W\|^{3}[A]_{\eta}(T)\right)(6.7)
$$

Proof. E(t) is defined in (5.6) and (4.19). From these equations it is seen that we need to estimate the following terms:

$$
\begin{aligned}
& \left(\alpha_{1}\right) \quad\left|R_{1 a}\right|=|A(0)|\left|\left(\beta \psi_{0}, e^{-i H_{0} t}\left(H_{0}-\lambda_{0}+\mu\right)^{-1} \mathbf{P}_{\mathbf{c}} \beta \psi_{0}\right)\right| \\
& \left(\alpha_{2}\right) \quad\left|R_{1 b}\right|=|A(0)| \mid \int_{0}^{t}\left(\beta \psi_{0}, e^{-i H_{0}(t-s)} e^{i\left(\mu-\lambda_{0}\right) s}\right. \\
& \left.\times\left(H_{0}-\lambda_{0}+\mu\right)^{-1} \partial_{s} A(s) \mathbf{P}_{\mathbf{c}} \beta \psi_{0}\right) d s \\
& \left(\alpha_{3}\right) \quad\left|R_{3}\right|=\frac{1}{2}|A(0)|\left|\left(\beta \psi_{0}, e^{-i H_{0} t}\left(H_{0}-\lambda_{0}-\mu-i 0\right)^{-1} \mathbf{P}_{\mathrm{c}} \beta \psi_{0}\right)\right| \\
& \left(\alpha_{4}\right) \quad\left|R_{4}\right|=\frac{1}{2} \mid \int_{0}^{t}\left(\beta \psi_{0}, e^{-i H_{0}(t-s)}\left(H_{0}-\lambda_{0}-\mu-i 0\right)^{-1}\right. \\
& \left.\times e^{-i\left(\lambda_{0}+\mu\right) s} \partial_{s} A(s) \mathbf{P}_{\mathrm{c}} \beta \psi_{0}\right) d s \\
& \left(\alpha_{5}\right) \quad\left|\left(\beta \psi_{0}, \phi_{0}\right)\right|=\left|\left(\beta \psi_{0}, e^{-i H_{0} \iota} \phi_{d}(0)\right)\right| \\
& \left(\alpha_{6}\right) \quad\left|\left(\beta \psi_{0}, \phi_{2}(t)\right)\right|=\left|\int_{0}^{t}\left(\beta \psi_{0}, e^{-i H_{0}(t-s)} \cos \mu s \mathbf{P}_{\mathbf{c}} \beta \phi_{d}(s) d s\right)\right|
\end{aligned}
$$

The estimates of $\left(\alpha_{j}\right)$ repeatedly use Lemma 6.1. Let $\eta \in[0, r-1]$.

Estimation of $\left(\alpha_{1}\right)$ :

$$
\begin{aligned}
\left|R_{1 a}\right| & \leqslant|A(0)|\left(w_{+} \beta \psi_{0}, w_{-} e^{-i H_{0} t}\left(H_{0}-\lambda_{0}+\mu\right)^{-1} \mathbf{P}_{\mathbf{c}} w_{-} w_{+} \beta \psi_{0}\right) \\
& \leqslant C|A(0)|\|W\|^{2}\langle t\rangle^{-r+1}
\end{aligned}
$$

by local decay estimates and our assumptions on $H_{0}$, since $\lambda_{0}-\mu$ is not in the spectrum of $H_{0}$. 
Estimation of $\left(\alpha_{2}\right)$. From (5.6) we have that

$$
\left|\partial_{s} A(s)\right| \leqslant C|| W|\|| A(s)|+| E(s) \mid
$$

since $\rho$ and $\Gamma$ are, respectively, linear and quadratic in $\|W\|$.

Applying Lemma 6.1 to $\left(\alpha_{2}\right)$ we then get

$$
\begin{aligned}
\left|R_{1 b}\right| \leqslant & |A(0)| \mid \int_{0}^{t}\left(w_{+} \beta \psi_{0}, w_{-} e^{-i H_{0}(t-s)}\right. \\
& \left.\times\left(H_{0}-\lambda_{0}+\mu\right)^{-1} \mathbf{P}_{\mathbf{c}} w_{-} \partial_{s} A(s) w_{+} \beta \psi_{0}\right) d s \mid \\
\leqslant & C\|W\|^{2}\langle t\rangle^{-\eta}\left(\mid\|W\|[A]_{\eta}(t)+[E]_{\eta}(t)\right)
\end{aligned}
$$

Estimation of $\left(\alpha_{3}\right)$. Since $\lambda_{0}+\mu \in \sigma\left(H_{0}\right)$, we use (2.4) together with the procedure used to estimate $\left(\alpha_{2}\right)$. The result is:

$$
\left|R_{3}\right| \leqslant C|A(0)||| W \mid \|^{2}\langle t\rangle^{-r+1}
$$

Estimation of $\left(\alpha_{4}\right)$. This term is estimated in a manner similar to $\alpha_{2}$ and $\alpha_{3}$ :

$$
\left|R_{4}\right| \leqslant c\|W\|^{2}\left\{\|W\|[A]_{\eta}(t)+[E]_{\eta}(t)\right\}\langle t\rangle^{-\eta}
$$

Estimation of $\left(\alpha_{5}\right)$. Since, by definition, $\phi_{\alpha}(0)=\mathbf{P}_{\mathbf{c}} \phi_{d}(0)$ we can apply local decay estimates for $e^{-i H_{0} t}$ to get

$$
\left|\left(\beta \psi_{0}, \phi_{0}(t)\right)\right| \leqslant C\|W\|\langle t\rangle^{-r+1}\left\|w_{+} \phi_{d}(0)\right\|
$$

Estimation of $\left(\alpha_{6}\right)$. Applying Lemma 6.1 as before we get, for $0 \leqslant t \leqslant T$,

$$
\left|\left(\beta \psi_{0}, \phi_{2}(t)\right)\right| \leqslant C\|W\|^{2}\langle t\rangle^{-\eta}\left[\phi_{d}\right]_{L D, \eta}(T)
$$

Using Proposition 6.1 to estimate $\left[\phi_{d}\right]_{L D, \eta}(t)$ in $(6.14)$, we get

$$
\left|\left(\beta \psi_{0}, \phi_{2}(t)\right)\right| \leqslant C\|W \mid\|^{2}\langle t\rangle{ }^{-\eta}\left\{\left\|w_{+} \phi_{d}(0)\right\|+\|W \mid\|[A]_{\eta}(t)\right\}
$$

Finally, combining the above estimates for $\left(\alpha_{j}\right), 1 \leqslant j \leqslant 6$, we can now bound $[E]_{\eta}(T)$ for any $\eta \in[0, r-1]$ as follows:

$$
\begin{aligned}
{[E]_{\eta}(t) \leqslant } & C\left\{\|W\|^{2}|A(0)|+\mid\|W\|\left\|w_{+} \phi_{d}(0)\right\|\right. \\
& \left.+\|W\|^{2}[E]_{\eta}(T)+\|W\| \|^{3}[A]_{\eta}(T)\right\}
\end{aligned}
$$

Since $\|W W \mid\|$ assumed to be small, Proposition 6.2 follows. 
We can now complete the proofs of our main results, Theorem 2.1 and Theorem 2.2. To prove the assertions concerning the infinite time behavior, the key is to establish local decay of $\phi_{d}$, in particular, the uniform boundedness of $\left[\phi_{d}\right]_{L D, r-1}(T)$. This will follow directly from Proposition 6.1 if we prove the uniform boundedness $[A]_{r-1}(T)$, or equivalently $[\tilde{A}]_{r-1}(T)$.

Proposition 6.3. There exists a constant $C_{*}$, depending on $\left\|\phi_{0}\right\|$, $\left\|w_{+} \phi_{0}\right\|,\|W\|, \Gamma$ and $r$, such that for any $T>0$

$$
[A]_{r-1}(T) \leqslant C_{*}
$$

Proof. We begin with the expansion of $A(t)$ given in Proposition 5.1. Multiplying (5.2) by $\langle t\rangle^{r-1}$, and taking the supremum over $0 \leqslant t \leqslant T$ we have:

$$
[A]_{r-1}(T) \leqslant\left(|A(0)| \Gamma^{-r+1}+\sup _{0 \leqslant \tau \leqslant 2 \Gamma^{-\alpha}}(\tau)^{r-1}\left|R_{A}(\tau)\right|+\sup _{2 \Gamma^{-\alpha} \leqslant \tau \leqslant T}(\tau)^{r-1}\left|R_{A}(\tau)\right|\right)
$$

The right hand side of $(6.18)$ is estimated using Proposition 5.1.

$$
\begin{aligned}
{[A]_{r-1}(T) \leqslant } & |A(0)| \Gamma^{-r+1}+D \Gamma^{-\alpha r}[E]_{0}\left(2 \Gamma^{-\alpha}\right) \\
& +C_{1} e^{-\Gamma^{-\delta}}[E]_{0}\left(2 \Gamma^{-\alpha}\right)+C \Gamma^{-1}[E]_{r-1}(T)
\end{aligned}
$$

Next, we apply Proposition 6.2 which yields:

$$
\begin{aligned}
{[A]_{r-1}(T) \leqslant } & |A(0)| \Gamma^{-r+1}+D \Gamma^{-\alpha r}[E]_{0}\left(2 \Gamma^{-\alpha}\right) \\
& +C_{1} e^{-\Gamma^{-\delta}}[E]_{0}\left(2 \Gamma^{-\alpha}\right) \\
& +C \Gamma^{-1}\left(|A(0)|\|W\|^{2}+\|W\|\left\|w_{+} \phi_{d}(0)\right\|\right. \\
& \left.+\|W\|^{3}[A]_{r-1}(T)\right)
\end{aligned}
$$

Note that by Proposition 6.2 and the simple bound:

$$
[A]_{r-1}(T) \leqslant C\langle T\rangle^{r-1}[A]_{0}(T) \leqslant C\langle T\rangle^{r-1}\left\|\phi_{0}\right\|
$$

$[E]_{0}\left(2 \Gamma^{-\alpha}\right)$ is bounded in terms of the initial data and $\|W\|$.

By hypothesis (H6), there is a positive constant $\theta_{0}$, such that

$$
\frac{\|W\|^{3}}{\Gamma} \leqslant \frac{\|W\|}{\theta_{0}}
$$


Therefore, for $\|W\| \|$ sufficiently small

$$
[A]_{r-1}(T) \leqslant C_{1 *}+C\|W\|\left[\phi_{d}\right]_{L D, r-1}(T)
$$

(Alternatively, we can obtain (6.22) by imposing that $\|W\|^{3} \Gamma^{-1}$ be sufficiently small.) Here, $C_{1 *}$ and subsequent constants depend on $\left\|\phi_{0}\right\|$, $\left\|w_{+} \phi_{0}\right\|,\|W\|, \Gamma$ and $r$.

Next, we use Proposition 6.1 to bound $\left[\phi_{d}\right]_{L D, r-1}(T)$, and we get

$$
[A]_{r-1}(T) \leqslant C_{2 *}+\|W\|^{2}[A]_{r-1}(T)
$$

Using smallness of $\|W\|$ again, we finally arrive at

$$
[A]_{r-1}(T) \leqslant C_{*}
$$

This completes the proof of Proposition 6.3 and therewith the $t \rightarrow \infty$ asymptotics asserted in Theorems 2.1 and 2.2.

Remark. This estimation procedure differs from one used in ref. 38 , where the cases $r-1$ large and $r-1$ small are treated differently.

It remains to prove the intermediate time estimate (2.12). The ingredients are contained in (6.7) and its proof. First, by (5.3)

$$
R_{A}(t) \leqslant C \int_{0}^{t} e^{-\Gamma(t-\tau)}|E(\tau)| d \tau
$$

Let $T_{0}$ denote an arbitrary fixed positive number. We estimate (6.24) for $t \in\left[0, T_{0} \Gamma^{-1}\right]$. We bound the exponential in the integrand by one (explicit integration would give something of order $\left.\Gamma^{-1}\right)$, and bound $|E(\tau)|$ by estimating the expressions $\left(\alpha_{j}\right), j=1, \ldots, 6$ in the proof of Proposition 6.2. First, the estimates of Proposition 6.2 for $\alpha_{1}, \alpha_{3}$ and $\alpha_{5}$ are useful as is. Integration of the bounds (6.8), (6.11) and (6.13) gives:

$$
\int_{0}^{t} e^{-\Gamma(t-\tau)}\left(\alpha_{j}\right) \leqslant C\|W\|\left\|w_{+} \phi(0)\right\|, \quad j=1,3,5
$$

To estimate the contributions of $\left(\alpha_{2}\right)$ and $\left(\alpha_{4}\right)$, first observe that by (6.9) and Proposition 6.2 with $\eta=0$

$$
\left|\partial_{s} A(s)\right| \leqslant C \mid\|W\|\left\|w_{+} \phi(0)\right\|
$$


Therefore, using local decay estimates we have:

$$
\begin{aligned}
\int_{0}^{t} e^{-\Gamma(t-\tau)}\left(\alpha_{j}\right) d \tau & \leqslant C T_{0} \Gamma^{-1}\|W\|^{3}\left\|w_{+} \phi(0)\right\| \\
& \leqslant\|W\|\left\|w_{+} \phi(0)\right\|, \quad j=2,4
\end{aligned}
$$

Finally, we come to the contribution of $\left(x_{6}\right)$. We rewrite $\left(x_{6}\right)$ as follows.

$$
\begin{aligned}
\left(\alpha_{6}\right) & =\left|\int_{0}^{t}\left(\beta e^{i H_{0}(t-s)} \mathbf{P}_{\mathbf{c}} \beta \psi_{0}, \cos \mu s \phi_{d}(s) d s\right)\right| \\
& =\left|\int_{0}^{t}\left(w_{+} \beta w_{+} \cdot w_{-} e^{i H_{0}(t-s)} \mathbf{P}_{\mathbf{c}} w_{-} \cdot w_{+} \beta \psi_{0}, \cos \mu s w_{-} \phi_{d}(s) d s\right)\right|
\end{aligned}
$$

Recall that by (4.9) $\phi_{d}=\phi_{0}+\phi_{1}+\phi_{2}$, where $\phi_{0}(t)=e^{-i H_{0} t} \phi_{d}(0)$. Using local decay estimates (H3), the contribution of the term $\phi_{0}(t)$ to $\left(\alpha_{6}\right)$ can be bounded by $C\|W\|^{2}\left\|w_{+} \phi_{d}(0)\right\|\langle\tau\rangle^{-r+1}$. Multiplication of this bound by $e^{-\Gamma(t-\tau)}$ and integration with respect to $t$ gives the bound $C\|W\|^{2}\left\|w_{+} \phi_{d}(0)\right\|$. To assess the contributions from $\phi_{1}+\phi_{2}$, note that local decay estimates (H3) imply

$$
\left\|w_{-}\left(\phi_{1}+\phi_{2}\right)\right\| \leqslant C\|W\|\left\|w_{+} \phi_{0}\right\|
$$

Putting together the contributions from $\phi_{0}$ and from $\phi_{1}+\phi_{2}$, we have:

$$
\int_{0}^{t} e^{-\Gamma(t-\tau)}\left(\alpha_{6}\right) d \tau \leqslant C\left(\|W\|^{2}\left\|w_{+} \phi_{d}(0)\right\|+\Gamma^{-1}\|W\|^{3}\right)
$$

The above estimates and (H6) imply (2.12). This concludes the proof of Theorem 2.2.

\section{APPENDIX A. GENERAL APPROACH TO LOCAL DECAY ESTIMATES}

Hypothesis (H3) for our main theorem is one requiring that our unperturbed operator, $H_{0}$, satisfy a suitable local decay estimates. It is remarked following this hypothesis that, in practice, the verification of (2.4) uses (2.5). In this section we give an outline to a very general approach to obtaining estimates of this type, based on a technique originating in the work of Mourre; ${ }^{(22)}$ see also ref. 25 . In the following general discussion we shall let $H$ denote self-adjoint operator on a Hilbert space, $\mathscr{H}$, keeping in 
mind that our application is to the unperturbed operator $H_{0}$. Let $E \in \sigma(H)$, and assume that an operator $A$ can be found such that $A$ is self-adjoint and $\mathscr{D}(A) \cap \mathscr{H}$ is dense in $\mathscr{H}$. Let $\Delta$ denote an open interval with compact closure. We shall use the notation:

$$
\left.\operatorname{ad}_{A}^{n}(H)=[\ldots[H, A], A], \ldots A\right]
$$

for the $\mathrm{n}$-fold commutator.

Assume the two conditions:

(M1) The operators

$$
\begin{aligned}
& g_{\Delta}(H)[H, A],[H, A] g_{A}(H) \quad \text { and } \\
& g_{\Delta}(H) \operatorname{ad}_{A}^{n}(H) g_{\Delta}(H), \quad 2 \leqslant n \leqslant N
\end{aligned}
$$

can all be extended to a bounded operator on $\mathscr{H}$.

(M2) Mourre estimate:

$$
g_{\Delta}(H) i[H, A] g_{\Delta}(H) \geqslant \theta g_{\Delta}(H)^{2}+K
$$

for some $\theta>0$ and compact operator, $K$.

Remark. The theorems below were orginally proved under more restrictive conditions than (M1). ${ }^{(5,8,22,25,34,36)}$ The more general results stated here can be proved using the approach to velocity bounds in ref. 12 .

Theorem A.1 (Mourre; see ref. 5, Theorem 4.9; ref. 2, Lemma 5.4). Assume conditions (M1)-(M2), with $N=2$. Then, in the interval $\Delta, H$ can only have absolutely continuous spectrum with finitely many eigenvalues of finite multiplicity. Moreover, the operator

$$
\langle A\rangle^{-1} g_{\Delta}(H)(H-z)^{-1}\langle A\rangle^{-1}
$$

is uniformly bounded in $z$, as an operator on $\mathscr{H}$. If $K=0$, then there are no eigenvalues in the interval $A$.

Theorem A.2 (Sigal-Soffer; see refs. 34, 8, 36, 11, and 12). Assume conditions (M1)-(M2) with $N \geqslant 2$ and $K=0$. Then, for all $\varepsilon>0$

$$
\left\|F\left(\frac{A}{t}<\theta\right) e^{-i H t} g_{A}(H) \psi\right\|_{2} \leqslant C\langle t\rangle^{-N+1+\varepsilon}\left\|\langle A\rangle^{N-1} \psi\right\|_{2}
$$


and therefore

$$
\left\|\langle A\rangle^{-\sigma} e^{-i H t} g_{\Delta}(H) \psi\right\|_{2} \leqslant C\langle t\rangle^{-\sigma}\left\|\langle A\rangle^{N-1} \psi\right\|_{2}
$$

for $\sigma<N-1$. Here, $F$ is a smoothed out characteristic function, and $F(A / t<\theta)$ is defined by the spectral theorem.

Remark. $\sigma$ is required to be smaller than $N / 2$ in refs. 8 and 34 and smaller than that in ref. 36. The above bound $\sigma<N-1$ is proved in ref. 12; see Theorem 1.1 and the remark below it.

Let $\Delta_{1}$ denote an open interval containing the closure of $\Delta$.

Corollary A.1. Assume that $\langle x\rangle^{-\sigma} g_{\Delta_{1}}(H)\langle A\rangle^{\sigma}$ is bounded. Then, in the above theorems we can replace the weight $\langle A\rangle^{-\sigma}$ by $\langle x\rangle^{-\sigma}$.

The strategy for using the above results to prove local decay estimates like that in (H4) is as follows. Then

$$
\begin{aligned}
&\left\|\langle x\rangle^{-\sigma} e^{-i H t} g_{\Delta}(H) \psi\right\|_{2} \\
&=\left\|\langle x\rangle^{-\sigma} g_{\Delta_{1}}(H) e^{-i H t} g_{\Delta}(H) \psi\right\|_{2} \\
&=\left\|\langle x\rangle^{-\sigma} g_{\Delta_{1}}(H)\langle A\rangle^{\sigma} \cdot\langle A\rangle^{-\sigma} e^{-i H t} g_{\Delta}(H) \psi\right\| \\
& \leqslant\left\|\langle x\rangle^{-\sigma} g_{A_{1}}(H)\langle A\rangle^{\sigma}\right\| \cdot\left\|\langle A\rangle^{-\sigma} e^{-i H t} g_{\Delta}(H) \psi\right\| \\
& \leqslant C\left\|\langle A\rangle^{-\sigma} e^{-i H t} g_{\Delta}(H) \psi\right\| \\
& \leqslant C_{1} \| F\left(\frac{A}{t}\langle\theta)\langle A\rangle^{-\sigma} e^{-i H t} g_{\Delta}(H) \psi \|_{2}\right. \\
&+C_{2}\left\|F\left(\frac{A}{t} \geqslant 0\right)\langle A\rangle^{-\sigma} e^{-i H t} g_{\Delta}(H) \psi\right\|_{2}
\end{aligned}
$$

Theorem A.2 is used to obtain the decay of the first term on the right hand side of (A.7), while we can replace $A$ by $\theta t$ in the second term.

\section{ACKNOWLEDGMENTS}

The authors wish to thank Eduard Kirr for helpful comments on the manuscript. AS and MIW are supported in part by grants from the National Science Foundation. 


\section{REFERENCES}

1. L. Alan and J. H. Eberley, Optical Resonance and Two Level Atoms (Dover, 1987).

2. V. Bach, J. Fröhlich, I. M. Sigal, and A. Soffer, Positive commutators and the spectrum of Pauli-Fierz Hamiltonians of atoms and molecules, 1997, preprint.

3. J. Carr, Applications of Center Manifold Theory (Springer-Verlag, New York, 1981).

4. J. Cooper and W. Strauss, Time periodic scattering of symmetric hyperbolic systems, J. Math. Anal. Appl. 122:444-452 (1987).

5. H. L. Cycon, R. G. Froese, W. Kirsch, and B. Simon, Schrödinger Operators with Applications to Quantum Mechanics and Global Geometry (Springer-Verlag, Berlin/Heidelberg/ New York, 1987).

6. V. Enss and K. Veselic, Bound states and propagation states for time dependent hamiltonians, Amn. Inst. H. Poincaré A 39:159-191 (1983).

7. A. Galindo and P. Pascual, Quantum Mechanics II (Springer, 1991).

8. C. Gérard and I. M. Sigal, Space-time picture of semiclassical resonances, Commun. Math. Phys. 145:281-328 (1992).

9. J. S. Howland, Stationary scattering theory for time-dependent Hamiltonians, Math. Ann. 207:315-335 (1974).

10. J. S. Howland, Scattering theory for Hamiltonians periodic in time, Indiana Math. J. 28:471-494 (1979).

11. W. Hunziker and I. M. Sigal, Time dependent scattering theory for N-body quantum systems, ETH-Zürich preprint (1997).

12. W. Hunziker, I. M. Sigal, and A. Soffer, Minimal escape velocities, 1998, preprint.

13. A. Jensen and T. Kato, Spectral properties of Schrödinger operators and time-decay of wave functions, Duke Math. J. 46:583-611 (1979).

14. H. R. Jauslin and J. L. Lebowitz, Generalized Floquet operator for quasiperiodically driven quantum systems, in Mathematical Physics X, K. Schmudgen, ed. (Springer, 1991, and cited ref.).

15. J.-L. Journé, A. Soffer, and C. Sogge, $L^{p} \rightarrow L^{p^{\prime}}$ estimates for the time dependent Schrödinger equation, Bull. AMS 23 (1990).

16. C.-A. Pillet and C. E. Wayne, Invariant manifolds for a class of dispersive, Hamiltonian partial differential equations, J. Diff. Eq. 141:310-326 (1997).

17. H. Kitada and $K$. Yajima, A scattering theory for time dependent long range potentials, Duke Math. J. 49:341-376 (1982).

18. H. Lamb, on a peculiarity of the wave-system due to the free vibrations of a nucleus in an extended medium, Proc. London Math. Soc. 32:208-211 (1900).

19. L. D. Landau and E. M. Lifshitz, Quantum Mechanics: Non-relativistic Theory, in Course of Theoretical Physics (Pergamon Press, Oxford, 1965), Vol. 3.

20. J. L. Lebowitz, Macroscopic laws, microscopic dynamics, time's arrow and Boltzmann's entropy, Phys. A 194(1-4):1-27 (1993).

21. P. D. Miller, A. Soffer, and M. I. Weinstein, Metastability of breather modes of time dependent potentials, in preparation.

22. E. Mourre, Absence of singular continuous spectrum for certain self-adjoint operators, Commun. Math. Phys. 78:391 (1981).

23. M. Murata, Rate of decay of local energy and spectral properties of elliptic operators, Jpn. J. Math. 6:77-127 (1980).

24. R. M. Pyke and I. M. Sigal, Nonlinear wave equations: Constraints on periods and exponential bounds for periodic solutions, Duke Math. J. 88 (1997).

25. P. Perry, I. M. Sigal, and B. Simon, Spectral analysis of N-body Schrödinger operators, Ann. Math. 114:519-567 (1981). 
26. R. Pyke, A. Soffer, and M. I. Weinstein, Stability of Kinks, in progress.

27. J. Rauch, Local decay of scattering solutions to Schrödinger's equation, Comm. Math. Phys. 61:149-168 (1978).

28. M. Reed and B. Simon, Methods in Modern Mathematical Physics, I. Functional Analysis (Academic Press, 1972).

29. M. Reed and B. Simon, Methods in Modern Mathematical Physics, IV. Analysis of Operator (Academic Press, 1978).

30. J. J. Sakurai, Advanced Quantum Mechanics (Addison Wesley, 1967).

31. T. Schonbek, Decay of solutions of Schrödinger equations, Duke Math. J. 46:203-213 (1979).

32. I. M. Sigal, Nonlinear wave and Schrödinger equations I. Instability of time-periodic and quasiperiodic solutions, Commun. Math. Phys. 153:297 (1993).

33. I. M. Sigal, General characteristics of nonlinear dynamics, in Spectral and Scattering Theory; Proceedings of the Taniguchi International Workshop, M. Ikawa, ed. (Marcel Dekker, New York/Basel/Hong Kong, 1994).

34. I. M. Sigal and A. Soffer, Local decay and velocity bounds for Quantum propagation, preprint (Princeton, 1988) (ftp://www.math.rutgers.edu/pub/soffer).

35. I. M. Sigal and A. Soffer, Asymptotic completeness for short range many body systems, Ann. Math. 126:35-108 (1987).

36. E. Skibsted, Propagation estimates for N-body Schrödinger operators, Commun. Math. Phys. 142:67-98 (1991).

37. A. Soffer and M. I. Weinstein, Dynamic theory of quantum resonances and perturbation theory of embedded eigenvalues, in Proceedings of Conference on Partial Differential Equations and Applications, University of Toronto, June, 1995, CRM Lecture Notes, P. Greiner, V. Ivrii, L. Seco, and C. Sulem, eds.

38. A. Soffer and M. I Weinstein, Time dependent resonance theory, to appear in Geometric and Functional Analysis.

39. A. Soffer and M. I. Weinstein, Resonances, radiation damping and instability in Hamiltonian nonlinear wave equations, to appear in Inventiones Mathematicae.

40. B. Vainberg, Scattering of waves in a medium depending periodically on time, Asterisque 210:327-340 (1992).

41. A. Vanderbauwhede and G. Iooss, Center manifold theory in infinite dimensions, Dynamics Reported 2 (1990).

42. J. M. G. Vilar and J. M. Rubi, Phys. Rev. Lett. 77:2863 (1996) and cited ref.

43. K. Yajima, Scattering theory for Schrödinger operators with potentials periodic in time, J. Math. Soc. Japan 29:729-743 (1977).

44. K. Yajima, Resonances for the AC-Stark effect, Commun. Math. Phys. 78:331-352 (1982).

45. K. Yajima, A multichannel scattering theory for some time dependent hamiltonians, charge transter problem, Commun. Math. Phys. 75:153-178 (1980). 